\title{
Raynaud's vector bundles and base points of the generalized Theta divisor
}

\author{
Georg Hein \\ July 10, 2006
}

\begin{abstract}
We study base points of the generalized $\Theta$-divisor on the moduli space of vector bundles on a smooth algebraic curve $X$ of genus $g$ defined over an algebraically closed field. To do so, we use the derived categories $\mathrm{D}^{\mathrm{b}}\left(\operatorname{Pic}^{0}(X)\right)$ and $\mathrm{D}^{\mathrm{b}}(\operatorname{Jac}(X))$ and the equivalence between them given by the Fourier-Mukai transform $\mathrm{FM}_{\mathcal{P}}$ coming from the Poincaré bundle. The vector bundles $P_{m}$ on the curve $X$ defined by Raynaud play a central role in this description. Indeed, we show that $E$ is a base point of the generalized $\Theta$-divisor, if and only if there exists a nontrivial homomorphism $P_{\operatorname{rk}(E) g+1} \rightarrow E$.
\end{abstract}

\section{Introduction}

Let $X$ be a smooth projective curve of genus $g \geq 1$ defined over an algebraically closed field of arbitrary characteristic. If $E$ is a sheaf on $X$ such that $h^{0}(X, E \otimes L)=0=h^{1}(X, E \otimes L)$ for a line bundle $L$ of degree zero, then it is an easy exercise to check that $E$ is a semistable vector bundle with slope $\mu(E):=\frac{\operatorname{deg}(E)}{\operatorname{rk}(E)}=g-1$. However, not for all semistable vector bundles $E$ of that slope does such a line bundle $L$ exist. Those stable vector bundles form the base locus of the generalized $\Theta$-divisor. Therefore, we say that a vector bundle $E$ has no $\Theta$-divisor if $H^{0}(E \otimes L) \neq 0$ for all line bundles $L$ of degree zero.

The first examples of such vector bundles were constructed by Raynaud in [10] giving one of the first applications of Fourier-Mukai transforms developed in [7]. He constructed a sequence of semistable vector bundles $P_{m}$ for $m \in \mathbb{N}$ having the property that $H^{0}(L \otimes$ $\left.P_{m}\right) \neq 0$ for all line bundles $L$ of degree zero.

A vector bundle $E$ with a nontrivial morphism $P_{m} \rightarrow E$ yields an example of a vector bundle without $\Theta$-divisor. Our aim is to show these are all examples. We show in theorem 25 that a vector bundle $E$ has no $\Theta$-divisor if and only if $\operatorname{Hom}\left(P_{m}, E\right) \neq 0$ for $m \gg 0$. In Theorem 37 we show that the condition on $m$ can be made effective. In Section 4 we define minimal vector bundles to be the minimal ones having no $\Theta$-divisor and show in Theorem 46 how they are related to ample divisors on the Picard group $\operatorname{Pic}^{0}(X)$. In section 5 we use the theory of spectral curves to show that we obtain Raynaud bundles $P_{m, R}$ having the property that for any vector bundle $E$ on $X$ the conditions $H^{0}(E \otimes F) \neq 0$ for all vector bundles $F$ of rank $R$ and degree zero is equivalent to $\operatorname{Hom}\left(P_{m, R}, E\right) \neq 0$. For more details see Theorem 52 . Finally, in the last section we provide some more applications. 
Acknowledgment. I would like to thank K. Altmann and D. Ploog for excellent discussions at their interesting university.

\section{The vector bundles $P_{m}$}

2.1 Notations. Let $\mathcal{P}_{X}$ be a Poincaré bundle on $X \times \operatorname{Pic}^{0}(X)$. This line bundle is the pull back of a Poincaré bundle $\mathcal{P}$ on $\operatorname{Jac}(X) \times \operatorname{Pic}^{0}(X)$. Here $\operatorname{Jac}(X)$ denotes the dual abelian variety of $\operatorname{Pic}^{0}(X)$ which is isomorphic to to the principally polarized abelian variety $\operatorname{Pic}^{0}(X)$. Let $x_{0}$ be a geometric point on $X$. We denote its image in $\operatorname{Jac}(X)$ also by $x_{0}$. With this point as zero $\operatorname{Jac}(X)$ becomes a group. We denote the map assigning an element its inverse by $[-1]_{\operatorname{Jac}(X)}$. The Poincaré bundle $\mathcal{P}$ becomes unique, when we require $\left.\mathcal{P}\right|_{\left\{x_{0}\right\} \times \operatorname{Pic}^{0}(X)} \cong \mathcal{O}_{\left\{x_{0}\right\} \times \operatorname{Pic}^{0}(X)}$. On $\operatorname{Pic}^{0}(X)$ we have the Theta divisor $\Theta_{\text {Pic }}:=\left\{[L] \in \operatorname{Pic}^{0}(X) \mid h^{1}\left(X, L \otimes L_{g-1}\right)>0\right\}$ where $L_{g-1}:=\mathcal{O}_{X}\left((g-1) x_{0}\right)$ is a fixed line bundle of degree $g-1$ on $X$. Resuming, we have the following commutative diagram of varieties and morphisms:

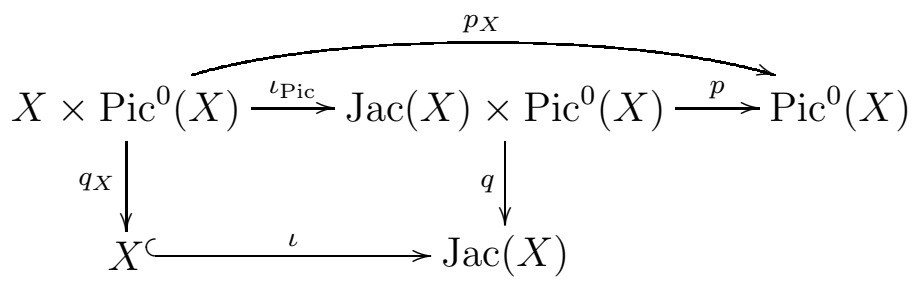

We will use the following line bundles

$$
\begin{array}{ll}
\mathcal{P} \in \operatorname{Pic}\left(\operatorname{Jac}(X) \times \operatorname{Pic}^{0}(X)\right) & \text { the Poincaré bundle } \\
\mathcal{P}_{X}=\iota_{\text {Pic }}^{*} \mathcal{P} & \text { the universal bundle on } X \times \operatorname{Pic}^{0}(X) \\
\mathcal{O}_{\text {Pic }}\left(\Theta_{\text {Pic }}\right) & \text { the Theta line bundle on the Picard group }
\end{array}
$$

2.2 Definition of the vector bundle $P_{m}$. Let $m$ be a positive integer. We define the vector bundle $P_{m}$ to be the following direct image sheaf:

$$
P_{m}:=\iota^{*}[-1]_{\mathrm{Jac}(X)}^{*} R^{g} q_{*}\left(\mathcal{P} \otimes p^{*} \mathcal{O}_{\mathrm{Pic}}\left(-m \cdot \Theta_{\mathrm{Pic}}\right)\right) .
$$

From Kodaira vanishing theorem and Serre duality we conclude the vanishing of the direct image sheaves $R^{i} q_{*}\left(\mathcal{P} \otimes p^{*} \mathcal{O}_{\text {Pic }}\left(-m \cdot \Theta_{\text {Pic }}\right)\right)$ for $i<g$. Therefore, we can use the Riemann-Roch theorem to calculate the numerical invariants of $P_{m}$.

2.3 Lemma. The vector bundle $P_{m}$ is semistable with numerical invariants

$$
\operatorname{rk}\left(P_{m}\right)=m^{g} \quad \operatorname{deg}\left(P_{m}\right)=g \cdot m^{g-1} \quad \mu\left(P_{m}\right)=\frac{g}{m} .
$$

Proof: This is a direct consequence of 3.1. in Raynaud's article [10]. The only difference is the application of $[-1]_{\mathrm{Jac}(X)}^{*}$ in our definition of $P_{m}$. Since $C$ and $[-1]_{\mathrm{Jac}(X)}^{*} C$ are 0 homologous cycles this morphism does not change the numerical invariants of the bundles.

2.4 Lemma. For any $m>0$ there exists a canonical surjection $P_{m+1} \longrightarrow P_{m}$.

Proof: Since $\left(\operatorname{Pic}^{0}(X), \Theta_{\text {Pic }}\right)$ is principally polarized, there exist (up to a scalar multiple) a unique morphism $\mathcal{O}_{\operatorname{Pic}^{0}(X)}\left(-(m+1) \Theta_{\mathrm{Pic}}\right) \stackrel{\psi}{\rightarrow} \mathcal{O}_{\operatorname{Pic}^{0}(X)}\left(-m \Theta_{\mathrm{Pic}}\right)$. The morphism $\psi$ is 
injective and its cokernel has support on the divisor $\Theta$. Thus, we obtain the canonical surjection on $\operatorname{Jac}(X)$

$$
R^{g} q_{*}\left(\mathcal{P} \otimes p^{*} \mathcal{O}_{\text {Pic }}\left(-(m+1) \cdot \Theta_{\text {Pic }}\right)\right) \longrightarrow R^{g} q_{*}\left(\mathcal{P} \otimes p^{*} \mathcal{O}_{\text {Pic }}\left(-m \cdot \Theta_{\text {Pic }}\right)\right)
$$

Pulling this surjection via $\iota^{*}[-1]_{\mathrm{Jac}(X)}^{*}$ back to $X$ yields the asserted surjection.

2.5 Theorem. For a coherent sheaf $F$ on $X$ we have the equivalence:

(For all $[L] \in \operatorname{Pic}^{0}(X)$ we have $\left.\operatorname{Hom}(L, F) \neq 0\right) \Leftrightarrow\left(\operatorname{Hom}\left(P_{m}, F\right) \neq 0\right.$ for all $\left.m \gg 0\right)$.

Proof: $\operatorname{Suppose} \operatorname{Hom}(L, F)=0$ for a line bundle $[L] \in \operatorname{Pic}^{0}(X)$. By semicontinuity this holds for all $[L] \in U \subset \operatorname{Pic}^{0}(X)$ for some open set $U$. Thus, the sheaf $p_{X *}\left(\mathcal{P}_{X} \otimes q_{X}^{*} F\right)$ is torsion. Since the direct image sheaf $p_{X *}\left(\mathcal{P}_{X} \otimes q_{X} F\right)$ is torsion free, we conclude that $p_{X *}\left(\mathcal{P}_{X} \otimes q_{X} F\right)=0$. Expressing this in terms of the Fourier-Mukai transform $\mathrm{FM}_{\mathcal{P}}: \mathrm{D}^{\mathrm{b}}(\operatorname{Jac}(X)) \rightarrow \mathrm{D}^{\mathrm{b}}\left(\operatorname{Pic}^{0}(X)\right)$ we obtain the equality $\mathrm{FM}_{\mathcal{P}}\left(\iota_{*} F\right)=G[-1]$ for a sheaf $G$ on $\operatorname{Pic}^{0}(X)$. Consequently, we have

$$
\operatorname{Hom}_{\mathrm{D}^{\mathrm{b}}\left(\operatorname{Pic}^{0}(X)\right)}\left(\mathcal{O}_{\operatorname{Pic}^{0}(X)}(-m \Theta), \operatorname{FM}_{\mathcal{P}}\left(\iota_{*} F\right)\right)=0
$$

for all integers $m$. The Fourier transform $\mathrm{FM}_{\mathcal{P}[g]}: \mathrm{D}^{\mathrm{b}}\left(\operatorname{Pic}^{0}(X)\right) \rightarrow \mathrm{D}^{\mathrm{b}}(\operatorname{Jac}(X))$ given by the kernel $\mathcal{P}[g]$ yields

$$
\operatorname{Hom}_{\mathrm{D}^{\mathrm{b}(\operatorname{Jac}(X))}}\left(\mathrm{FM}_{\mathcal{P}[g]}\left(\mathcal{O}_{\operatorname{Pic}^{0}(X)}(-m \Theta)\right),[-1]^{*} \iota_{*} F\right)=0 .
$$

Thus, we have $\operatorname{Hom}_{\mathrm{D}^{\mathrm{b}}(X)}\left(\iota^{*}[-1]^{*} \mathrm{FM}_{\mathcal{P}[g]}\left(\mathcal{O}_{\operatorname{Pic}^{0}(X)}(-m \Theta)\right), F\right)=0$. By the definition of $P_{m}$ this equals $\operatorname{Hom}_{\mathcal{O}_{X}}\left(P_{m}, F\right)=0$ for all integers $m \geq 1$.

If $\operatorname{Hom}(L, F) \neq 0$ for all $[L] \in \operatorname{Pic}^{0}(X)$, then, we have $F^{0}:=p_{X *}\left(\mathcal{P}_{X} \otimes q_{X} F\right)$ is a nontrivial coherent sheaf on $\operatorname{Pic}^{0}(X)$. Thus, for $m \gg 0$ we have $F^{0}(m \Theta)$ has global sections, or equivalently $\operatorname{Hom}\left(\mathcal{O}_{\operatorname{Pic}^{0}(X)}(-m \Theta), F^{0}\right) \neq 0$. We conclude

$$
\operatorname{Hom}_{\mathrm{D}^{\mathrm{b}}\left(\operatorname{Pic}^{0}(X)\right)}\left(\mathcal{O}_{\operatorname{Pic}^{0}(X)}(-n \Theta), \operatorname{FM}_{\mathcal{P}}\left(\iota_{*} F\right)\right) \neq 0 .
$$

As before, this yields $\operatorname{Hom}_{\mathcal{O}_{X}}\left(P_{m}, F\right) \neq 0$ for $m \gg 0$.

\section{Semistable vector bundles without $\Theta$-divisor}

3.1 Preliminaries. From 311 to Corollary 35 in this section $E$ denotes a vector bundle of rank $r$ and the property that $\chi(F) \leq 0$ for all subsheaves $F \subset E$. The last condition is equivalent to $\mu_{\max }(E) \leq g-1$. Furthermore, we assume that $E$ has no $\Theta$-divisor. Thus, the $\mathcal{O}_{\operatorname{Pic}^{0}(X)}{ }^{\text {-sheaves }} \operatorname{FM}_{\mathcal{P}}^{0}(E):=R^{0} p_{X *}\left(\mathcal{P} \otimes q_{X}^{*} E\right)$ and $\operatorname{FM}_{\mathcal{P}}^{1}(E):=R^{1} p_{X *}\left(\mathcal{P} \otimes q_{X}^{*} E\right)$ have positive rank. Let $D=P_{1}+P_{2}+\ldots+P_{g-1}$ be a reduced divisor of degree $g-1$ on $X$. Applying the functor $R^{\bullet} p_{X *}\left(\mathcal{P} \otimes q_{X}^{*}-\right)$ to the short exact sequence $0 \rightarrow E(-D) \rightarrow E \rightarrow$ $\mathcal{O}_{D}^{\oplus r} \rightarrow 0$ gives a long exact sequence

$$
\mathrm{FM}_{\mathcal{P}}^{0}(E(-D)) \rightarrow \mathrm{FM}_{\mathcal{P}}^{0}(E) \rightarrow \mathrm{FM}_{\mathcal{P}}^{0}\left(\mathcal{O}_{D}^{\oplus r}\right) \rightarrow \mathrm{FM}_{\mathcal{P}}^{1}(E(-D)) \rightarrow \mathrm{FM}_{\mathcal{P}}^{1}(E) \rightarrow \mathrm{FM}_{\mathcal{P}}^{1}\left(\mathcal{O}_{D}^{\oplus r}\right)
$$

of coherent sheaves on $\operatorname{Pic}^{0}(X)$.

3.2 Lemma. The sheaves $\mathrm{FM}_{\mathcal{P}}^{0}(E(-D))$ and $\mathrm{FM}_{\mathcal{P}}^{1}\left(\mathcal{O}_{D}^{\oplus r}\right)$ are zero. The direct image sheaf $\mathrm{FM}_{\mathcal{P}}^{0}\left(\mathcal{O}_{D}^{\oplus r}\right)$ is isomorphic to a direct sum of $r(g-1)$ line bundles numerically equivalent to $\mathcal{O}_{\operatorname{Pic}^{0}(X)}$. 
Proof: Let $L$ be a line bundle of degree 0 . If $h^{0}(E(-D) \otimes L)>0$, then we have a non trivial morphisms $L^{\vee} \rightarrow E(-D)$. Since $\mu_{\max }(E(-D)) \leq 0$ this implies that $\mu_{\max }(E(-D))=0$ and that $L^{\vee}$ is is a direct summand of the graduated object associated to the subbundle $\left.E_{\max } \subset E(-D)\right)$ of maximal slope. We deduce, that there exist at most $r$ line bundles $L \in \operatorname{Pic}^{0}(X)$ with $h^{0}(E(-D) \otimes L)>0$. Consequently, the torsion free sheaf $\operatorname{FM}_{\mathcal{P}}^{0}(E(-D))$ is zero.

Since the support of $\mathcal{O}_{D}$ is zero dimensional, there are no higher direct images. In particular, $\operatorname{FM}_{\mathcal{P}}^{1}\left(\mathcal{O}_{D}^{\oplus r}\right)=0 . \quad D$ is a reduced divisor, thus $\mathcal{O}_{D}=\oplus_{i=1}^{g-1} k\left(P_{i}\right)$. Thus, $\operatorname{FM}_{\mathcal{P}}^{0}\left(\mathcal{O}_{D}^{\oplus r}\right)=\left(\oplus_{i=1}^{g-1} \operatorname{FM}_{\mathcal{P}}^{0}\left(k\left(P_{i}\right)\right)\right)^{\oplus r}$.

3.3 Lemma. There exists a direct sum $\bigoplus_{k=0}^{-\chi(E)} M_{k}$ of line bundles numerically equivalent to $\mathcal{O}_{\mathrm{Pic}^{0}(X)}$ and a commutative diagram with injective vertical arrows

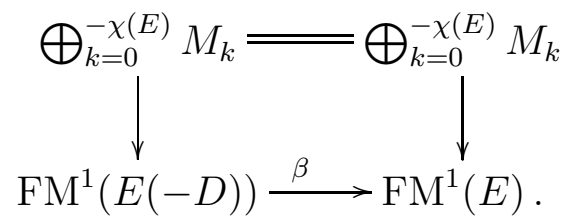

Proof: Let $M$ be a line bundle on $X$ of degree $N$ for some $N \gg 0$. By Serre duality and stability we conclude $H^{1}(E(-D) \otimes M \otimes L)=0$ for all line bundles $[L] \in \operatorname{Pic}^{0}(X)$. This is equivalent to $\operatorname{FM}_{\mathcal{P}}^{1}(E(-D) \otimes M)=0$. Taking a reduced section of $M$ and tensorizing with $E(-D)$ we obtain a short exact sequence

$$
0 \rightarrow E(-D) \rightarrow E(-D) \otimes M \rightarrow \bigoplus_{i=1}^{N} k\left(P_{i}\right)^{\oplus r} \rightarrow 0
$$

Applying the Fourier-Mukai transform $\mathrm{FM}_{\mathcal{P}}$ again, yields the exact sequence

$$
\mathrm{FM}_{\mathcal{P}}^{0}\left(\bigoplus_{i=1}^{N} k\left(P_{i}\right)^{\oplus r}\right) \rightarrow \mathrm{FM}_{\mathcal{P}}^{1}(E(-D)) \rightarrow 0=\mathrm{FM}_{\mathcal{P}}^{1}(E(-D) \otimes M)
$$

As seen in Lemma 32 the sheaf $\mathrm{FM}_{\mathcal{P}}^{0}\left(\bigoplus_{i=1}^{N} k\left(P_{i}\right)^{\oplus r}\right)$ is a direct sum of numerically trivial line bundles. Again by Lemma 32 we obtain a sequence of surjections

$$
\operatorname{FM}_{\mathcal{P}}^{0}\left(\bigoplus_{i=1}^{N} k\left(P_{i}\right)^{\oplus r}\right) \rightarrow \operatorname{FM}_{\mathcal{P}}^{1}(E(-D)) \rightarrow \operatorname{FM}_{\mathcal{P}}^{1}(E)
$$

This implies the assertion because the rank of $\operatorname{FM}_{\mathcal{P}}^{1}(E)$ is at least $1-\chi(E)$ by assumption.

3.4 Lemma. For a positive integer $m$ we have the three equalities

$$
\begin{gathered}
h^{0}\left(\bigoplus_{k=0}^{-\chi(E)} M_{k}(m \Theta)\right)=(1-\chi(E)) m^{g} \quad h^{0}\left(\mathrm{FM}^{0}\left(\mathcal{O}_{D}^{\oplus r}\right)(m \Theta)\right)=(g-1) r m^{g} \\
\quad \text { and } \quad h^{0}\left(\operatorname{FM}_{\mathcal{P}}^{1}(E(-D))(m \Theta)\right)=\left(g-1+\frac{g}{m}-\frac{\chi(E)}{r}\right) r m^{g} .
\end{gathered}
$$


Proof: The proofs of the statement for $h^{0}\left(\bigoplus_{k=0}^{-\chi(E)} M_{k}(m \Theta)\right)$ and $h^{0}\left(\mathrm{FM}^{0}\left(\mathcal{O}_{D}^{\oplus r}\right)(m \Theta)\right)$ are the same by Lemma 32 and follow by basic facts (see III.16 in Mumford's book [8]) on the cohomology of line bundles on abelian varieties.

Consider the vector bundle bundle $F_{m}:=\mathcal{P} \otimes q_{X}^{*}(E(-D)) \otimes p_{X}^{*} \mathcal{O}(m \Theta)$ on $X \times \operatorname{Pic}^{0}(X)$. The projection formula gives that $R^{i} p_{X *} F_{m}=\mathrm{FM}_{\mathcal{P}}^{i}(E(-D))(m \Theta)$. Since $p_{X}$ has fiber dimension one, and $\operatorname{FM}_{\mathcal{P}}^{0}(E(-D))=0$ by Lemma 32 we obtain by the Leray spectral sequence $H^{0}\left(F_{m}\right)=0$ and the isomorphism $H^{1}\left(F_{m}\right) \cong H^{0}\left(\operatorname{FM}_{\mathcal{P}}^{1}(E(-D))(m \Theta)\right)$.

Next we apply the Leray spectral sequence to $q_{X}$ and the global section functor. It is shown in [7] that $q_{X *}\left(\mathcal{P} \otimes p_{X}^{*} \mathcal{O}(m \Theta)\right)$ is the dual of $P_{m}$. Thus, by the projection formula $q_{X *}\left(F_{m}\right)=P_{m}^{\vee} \otimes E(-D)$, and $R^{i} q_{X *}\left(F_{m}\right)=0$ for all $i>0$. We deduce from the spectral sequence that $H^{0}\left(F_{m}\right)=H^{0}\left(P_{m}^{\vee} \otimes E(-D)\right)$ and $H^{1}(F) \cong H^{1}\left(P_{m}^{\vee} \otimes E(-D)\right)$. Putting the result together we find

$$
H^{0}\left(P_{m}^{\vee} \otimes E(-D)\right)=0 \quad H^{1}\left(P_{m}^{\vee} \otimes E(-D)\right) \cong H^{0}\left(\mathrm{FM}_{\mathcal{P}}^{1}(E(-D))\right) .
$$

Thus, $h^{0}\left(\mathrm{FM}_{\mathcal{P}}^{1}(E(-D))\right)=h^{1}\left(P_{m}^{\vee} \otimes E(-D)\right)=-\chi\left(P_{m}^{\vee} \otimes E(-D)\right)$. However, the last number can be directly computed by the Riemann-Roch theorem for curves.

3.5 Corollary. For $m>r \cdot g$ we have $h^{0}\left(\operatorname{FM}_{\mathcal{P}}^{0}(E)(m \Theta)\right)>0$.

Proof: From 32 and 33 we obtain the following diagram with exact row

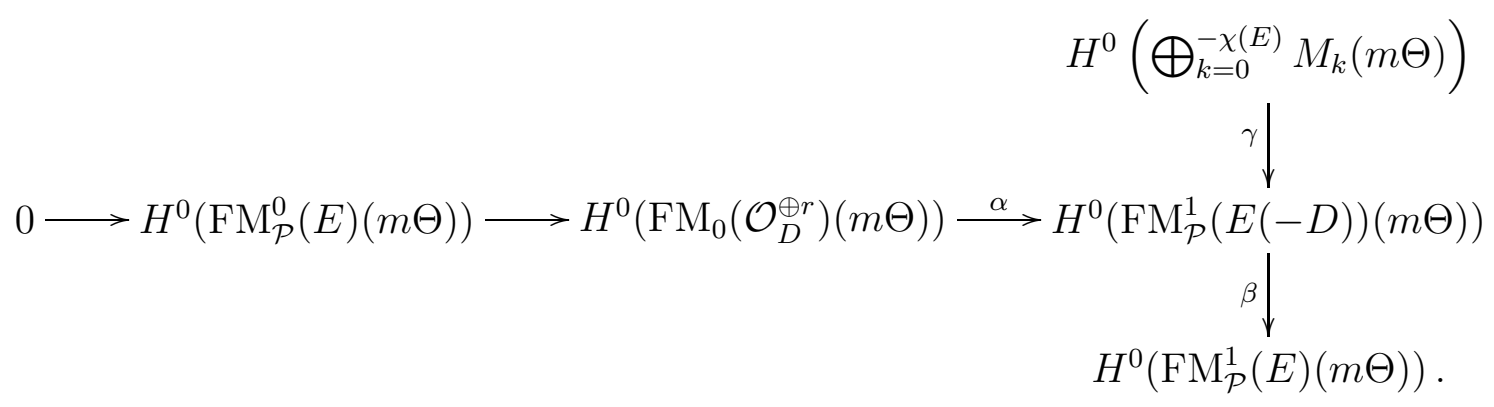

Furthermore, we have that $\beta \circ \gamma$ and $\gamma$ are injective by Lemma 33, and $\beta \circ \alpha=0$ from the long exact sequence before Lemma 32. Therefore the dimension of the image of $\alpha$ is at most $h^{0}\left(\operatorname{FM}_{\mathcal{P}}^{1}(E(-D))(m \Theta)\right)-h^{0}\left(\bigoplus_{k=0}^{-\chi(E)} M_{k}(m \Theta)\right)$. This number is known by Lemma 34 and by the assumption on $m$ strictly smaller than $h^{0}\left(\mathrm{FM}_{0}\left(\mathcal{O}_{D}^{\oplus r}\right)(m \Theta)\right)$.

3.6 Lemma. If $E^{\prime}$ is a vector bundle of with $\chi\left(E^{\prime}\right)>0$, and $r k\left(E^{\prime}\right) \leq r$, then for all $m>r \cdot g$ we have $\operatorname{Hom}\left(P_{m}, E^{\prime}\right) \neq 0$.

Proof: We have that the slope of $E^{\prime}$ is at least $\frac{1}{r}+(g-1)$. The condition on $m$ implies that the slope of $E^{\prime} \otimes P_{m}^{\vee}$ is strictly greater than $g-1$. Thus, $\chi\left(E^{\prime} \otimes P_{m}^{\vee}\right)>0$. This implies $0<\chi\left(E^{\prime} \otimes P_{m}^{\vee}\right) \leq h^{0}\left(E^{\prime} \otimes P_{m}^{\vee}\right)=\operatorname{dim}\left(\operatorname{Hom}\left(P_{m}, E^{\prime}\right)\right)$.

3.7 Theorem. Let $E$ be a vector bundle on $X$ of rank $r$. For any $m>r \cdot g$ we have the equivalence

$$
\text { E has a } \Theta \text {-divisor } \Leftrightarrow \operatorname{Hom}\left(P_{m}, E\right)=0 \text {. }
$$

Proof: If $E$ has a $\Theta$-divisor, then the claim follows from Theorem 25 and Lemma 24. Let us assume that $E$ has no $\Theta$-divisor. If for all $E^{\prime} \subset E$ we have $\chi\left(E^{\prime}\right) \leq 0$, then we are done by Corollary 35] and the identification of $\operatorname{Hom}\left(P_{m}, E\right)$ with $H^{0}\left(\mathrm{FM}_{\mathcal{P}}^{0}(E)(m \Theta)\right)$ given in Theorem 25. If not, then $E$ contain a subsheaf $E^{\prime}$ of positive Euler characteristic. In this case we use the above Lemma 36. 


\section{Minimal quotients of Raynaud's bundles}

\subsection{Minimal bundles}

$4.1 \chi$-small vector bundles. We have seen in Lemma 36 that for a sheaf $E$ on $X$ of positive Euler characteristic $\chi(E)$ we have morphisms from $P_{m}$ to $E$ for $m>g \cdot \operatorname{rk}(E)$. This implies, that for a vector bundle $E$ containing a vector bundle $E^{\prime}$ of positive Euler characteristic there are morphisms from $P_{m}$ to $E$ for $m \gg 0$. Therefore, the existence of morphisms from $P_{m}$ to vector bundles which do not contain any subsheaf of positive Euler characteristic has to be investigated. We call a sheaf $E$ which does not contain a subsheaf of positive Euler characteristic a $\chi$-small sheaf. This implies that $E$ is a vector bundle. Semistable vector bundles of slope $\leq g-1$ are examples of $\chi$-small bundles. $\chi$-smallness can be expressed in terms of the Harder-Narasimhan filtration $0 \subset E_{1} \subset E_{2} \subset \ldots \subset E$ of $E$ by the condition $\mu\left(E_{1}\right) \leq g-1$.

We frequently will use the obvious fact, that subsheaves of $\chi$-small vector bundles are $\chi$-small too.

4.2 Morphisms from $P_{m}$ to $\chi$-small bundles. Let $X$ be a curve of genus $g \geq 2$. Since $P_{2}$ is $\chi$-small, we have morphisms from $P_{m}$ to $P_{2}$ for all $m \geq 2$ by Lemma 24. We consider the following set of integers

$$
N:=\left\{\begin{array}{l|l}
\operatorname{rk}(E) & \begin{array}{l}
E \text { is a } \chi \text {-small vector bundle, } \\
\operatorname{Hom}\left(P_{m}, E\right) \neq 0 \text { for } m \gg 0
\end{array}
\end{array}\right\}
$$

By the above remark $2^{g}=\operatorname{rk}\left(P_{2}\right) \in N$. Thus, $N \neq \emptyset$. If $r \in N$, then there exists a $\chi$-small vector bundle $E$ of rank $r$ with $\operatorname{Hom}\left(P_{m}, E\right) \neq 0$. However, $E \oplus \mathcal{O}_{X}$ is $\chi$-small too, and $\operatorname{Hom}\left(P_{m}, E\right)$ is embedded into $\operatorname{Hom}\left(P_{m}, E \oplus \mathcal{O}_{X}\right)$. Eventually, we conclude $r+1 \in N$. If $r_{-}$is the minimal element of $N$, then we have seen, that $N=\left\{r_{-}, r_{-}+1, \ldots\right\}$. Of course, the number $r_{-}$depends on the curve $X$.

By definition we have that a semistable vector bundle $E$ on $X$ with $\chi(E)=0$ and $\operatorname{rk}(E)<r_{-}$is not a base point for the generalized $\Theta$-divisor.

4.3 Lemma. If $E$ is a $\chi$-small vector bundle of rank $r_{-}$on $X$ with $\operatorname{Hom}\left(P_{m}, E\right) \neq 0$ for $m \gg 0$, then there exists no non trivial homomorphism to a $\chi$-small vector bundle $E^{\prime \prime}$ of smaller rank. Moreover, $E$ is stable.

Proof: Suppose there exists a non trivial homomorphisms $E \stackrel{\alpha}{\rightarrow} E^{\prime \prime}$ to a $\chi$-small vector bundle $E^{\prime \prime}$ of $\operatorname{rank} \operatorname{rk}\left(E^{\prime \prime}\right)<r k(E)=r_{-}$. Since the image $\operatorname{im}(\alpha)$ is $\chi$-small too, we can assume that $\alpha$ is surjective. Setting $E^{\prime}=\operatorname{ker}(\alpha)$ we obtain another $\chi$-small vector bundle. Thus,

$$
0 \rightarrow E^{\prime} \rightarrow E \stackrel{\alpha}{\rightarrow} E^{\prime \prime} \rightarrow 0
$$

is a short exact sequence of $\chi$-small vector bundles. The minimality of $r_{-}$implies that $\operatorname{Hom}\left(P_{m}, E^{\prime}\right)=0=\operatorname{Hom}\left(P_{m}, E^{\prime \prime}\right)$. This implies $\operatorname{Hom}\left(P_{m}, E\right)=0$ which contradicts our assumption.

Suppose $E$ is not semistable. Take the first subsheaf $E_{1} \subset E$ of the Harder-Narasimhan filtration of $E$. $E_{1}$ and $E / E_{1}$ are $\chi$-small of lower rank which is a contradiction. If $E$ is semistable but not stable, then there exists a stable subbundle $E_{1} \subset E$ of the same slope. As before, $E_{1}$ and $E / E_{1}$ are $\chi$-small. Thus, we conclude the stability of $E$.

4.4 Minimal bundles. We call a vector bundle $E$ on the curve $X$ a minimal bundle when it satisfies the conditions 
(i) $\operatorname{Hom}\left(P_{m}, E\right) \neq 0$ for $m \gg 0$, and

(ii) $\operatorname{Hom}\left(P_{m}, E^{\prime}\right)=0$ for all proper subsheaves $E^{\prime} \varsubsetneqq E$.

By Theorems 25] and 37 the following conditions are equivalent to (i):

(i-a) $\quad H^{0}(E \otimes L) \neq 0$ for all $[L] \in \operatorname{Pic}^{0}(X)$,

(i-b) $\operatorname{Hom}(L, E) \neq 0$ for all $[L] \in \operatorname{Pic}^{0}(X)$,

(i-c) $\operatorname{Hom}\left(P_{m}, E\right) \neq 0$ for all $m>g \operatorname{rk}(E)$.

(i-d) $\operatorname{Hom}\left(P_{m}, E\right) \neq 0$ for one $m>g r k(E)$.

4.5 Lemma. If $E$ is a sheaf on $X$ with $\operatorname{Hom}\left(P_{m}, E\right) \neq 0$ for $m \gg 0$, then there exists a minimal subsheaf $F \subset E$.

Proof: We take an integer $m>\operatorname{grk}(E)$. For all $\phi \in \operatorname{Hom}\left(P_{m}, E\right)$ the vector bundle $F_{\phi}:=\operatorname{im}(\phi)$ fulfills $0 \leq \operatorname{rk}\left(F_{\phi}\right) \leq \operatorname{rk}(E)$, and $\mu\left(P_{m}\right) \leq \mu\left(F_{\phi}\right) \leq \mu\left(E_{1}\right)$ where $E_{1}$ is the sheaf from 42. Consequently, the Hilbert polynomials of the sheaves $\left\{F_{\phi}\right\}_{\phi \in \operatorname{Hom}\left(P_{m}, E\right)}$ form a finite set. Taking $F:=F_{\phi}$ with minimal possible Hilbert polynomial we obtain the desired subsheaf by Theorem 37.

The subsheaf $F$ of the above lemma is not unique. However, to understand sheaves $E$ with $\operatorname{Hom}\left(P_{m}, E\right) \neq 0$ it is convenient to study the minimal ones. This is what we do next.

\subsection{The structure of minimal bundles}

4.6 Theorem. Let $E$ be a minimal vector bundle on a smooth projective curve $X$ of genus g. Then there exists an ample divisor $D=D(E)$ on $\operatorname{Pic}^{0}(X)$ such that $\operatorname{FM}_{\mathcal{P}}^{0}\left(i_{*} E\right) \cong$ $\mathcal{O}_{\mathrm{Pic}^{0}(X)}(-D)$, and the vector bundle $P_{D}:=\iota^{*}[-1]_{\mathrm{Jac}(X)}^{*} R^{g} q_{*}\left(\mathcal{P} \otimes p^{*} \mathcal{O}_{\operatorname{Pic}^{0}(X)}(-D)\right)$ admits a unique surjection to $E$.

Proof: The proof of this theorem will be a consequence of the results in lemma 48 413. Throughout this subsection $E$ is a fixed minimal bundle.

4.7 Lemma. Each nonzero morphism $\psi: P_{m} \rightarrow E$ to the minimal bundle $E$ is surjective. Proof: If $\psi: P_{m} \rightarrow E$ is not surjective, then the image of $\psi$ is a vector bundle with a morphism from $P_{m}$ to it. This contradicts the minimality of $E$.

4.8 Lemma. For the minimal bundle $E$ the sheaf $\operatorname{FM}_{\mathcal{P}}^{0}(E)$ is an ideal sheaf $\mathcal{J}_{Z}$.

Proof: We consider a surjection $E \rightarrow k\left(x_{0}\right)$, and let $E^{\prime}$ be the kernel. We obtain an exact sequence

$$
0 \rightarrow \operatorname{FM}_{\mathcal{P}}^{0}\left(E^{\prime}\right) \rightarrow \operatorname{FM}_{\mathcal{P}}^{0}(E) \rightarrow \operatorname{FM}_{\mathcal{P}}^{0}\left(k\left(x_{0}\right)\right)=\mathcal{O}_{\text {Pic }}
$$

However, the minimality of $E$ implies that $\operatorname{FM}_{\mathcal{P}}^{0}\left(E^{\prime}\right)=0$, and $\operatorname{FM}_{\mathcal{P}}^{0}(E) \neq 0$.

4.9 The divisor $D$. The ideal sheaf $\mathcal{J}_{Z}$ can be decomposed as $\mathcal{O}_{\text {Pic }}(-D) \otimes \mathcal{J}_{Z^{\prime}}$ where $D$ is a divisor and $Z^{\prime}$ is a subscheme of codimension greater than one. This decomposition corresponds to the decomposition $Z=D \cup Z^{\prime}$ of $Z$ into its irreducible components of codimension one and those of greater codimension.

4.10 Lemma. $D$ is an ample divisor.

Proof: First $D$ is an effective divisor. Thus, the linear system $|2 D|$ is base point free. Thus, taking the Stein factorization of the morphism defined by the linear system $|2 D|$, we obtain a surjective morphism $\pi: \mathrm{Pic}^{0}(X) \rightarrow Y$ of projective varieties with connected fibers. Let $T$ be the subscheme $\pi^{-1}(\pi(0))$ with the reduced scheme structure. $D$ is ample if and only if $\pi$ is an isomorphism. Thus, we have the obvious implication ( $D$ is ample) $\Rightarrow$ 
( $T$ is a point). The converse implication holds too. Indeed, assume that $T$ is a point. If all fibers of $\pi$ have dimension zero, then $D$ is ample. Suppose that $C$ is curve in $\operatorname{Pic}^{0}(X)$ contained in a fiber of $\pi$. Thus, $\operatorname{deg}\left(\left.\mathcal{O}(D)\right|_{C}\right)=0$. This holds true for all translates of the curve $C$. Let $C^{\prime}$ be a translate of $C$ passing through 0 , then $C^{\prime}$ must be contained in $T$ which is a contradiction. Concluding, we have ( $D$ is ample $) \Leftrightarrow(T$ is a point $)$.

$T$ is by definition a closed subscheme and closed under the group operation of $\operatorname{Pic}^{0}(X)$. Hence, the embedding $\tau: T \rightarrow \operatorname{Pic}^{0}(X)$ is a morphism of abelian varieties. We obtain a surjection $\tau^{*}: \operatorname{Pic}^{0}\left(\operatorname{Pic}^{0}(X)\right) \rightarrow \operatorname{Pic}^{0}(T)$ by $[L] \mapsto\left[\left.(\mathcal{O}(2 D) \otimes L)\right|_{T}\right]$.

Let $[L] \in \operatorname{Pic}^{0}\left(\operatorname{Pic}^{0}(X)\right)$ be a line bundle on $\operatorname{Pic}^{0}(X)$ with $h^{0}(\mathcal{O}(2 D) \otimes L)>0$. Since, $\mathcal{O}(2 D) \otimes L$ is numerically trivial on the fibers of $\pi$, the existence of a global section implies that it is trivial on the generic fiber. Hence, by the seesaw theorem (see [8], p. 54) it is trivial on all fibers, in particular it is trivial on $T$. Thus, the set $\{[L] \in$ $\left.\operatorname{Pic}^{0}\left(\operatorname{Pic}^{0}(X)\right) \mid h^{0}(\mathcal{O}(2 D) \otimes L)>0\right\}$ is contained in the subgroup $\operatorname{ker}\left(\tau^{*}\right)$.

Let $Q \in X$ be a geometric point, which maps under $\iota: X \rightarrow \operatorname{Pic}^{0}\left(\operatorname{Pic}^{0}(X)\right)$ to the line bundle $L_{Q}$. As in the proof of lemma 48, we conclude that there exists an injection $\mathrm{FM}_{\mathcal{P}}^{0}(E) \rightarrow L_{Q}$. Since $\operatorname{Hom}\left(\mathrm{FM}_{\mathcal{P}}^{0}(E), L_{Q}\right)=\operatorname{Hom}\left(\mathcal{O}(-D), L_{Q}\right)=H^{0}\left(L_{Q}(D)\right)$, we conclude that the line bundle $L_{Q}(D)$ has a global section. Consequently, for any two points $Q, Q^{\prime} \in X$ the line bundle $L_{Q} \otimes L_{Q^{\prime}}(2 D)$ has a global section.

However, $\operatorname{Pic}^{0}\left(\operatorname{Pic}^{0}(X)\right)$ is the smallest subgroup of $\operatorname{Pic}^{0}\left(\operatorname{Pic}^{0}(X)\right)$ which contains the image of $\iota: X \rightarrow \operatorname{Pic}^{0}\left(\operatorname{Pic}^{0}(X)\right)$. Thus, the kernel of $\tau^{*}$ is $\operatorname{Pic}^{0}\left(\operatorname{Pic}^{0}(X)\right)$ itself. This implies that $\operatorname{dim}(T)=0$. Hence, the assertion of the lemma holds.

4.11 Lemma. The subscheme $Z^{\prime}$ of 4 [ is empty. Hence, $\mathcal{J}_{Z}=\mathcal{O}_{\operatorname{Pic}^{0}(X)}(-D)$.

Proof: We start with the short exact sequence on $\operatorname{Pic}^{0}(X)$ :

$$
\left.0 \rightarrow \mathcal{J}_{Z} \rightarrow \mathcal{O}_{\operatorname{Pic}^{0}(X)}(-D) \rightarrow \mathcal{O}_{\operatorname{Pic}^{0}(X)}(-D)\right|_{Z^{\prime}} \rightarrow 0
$$

and the resulting long exact sequence

$$
\ldots \rightarrow \mathrm{FM}_{\mathcal{P}}^{i-1}\left(\left.\mathcal{O}_{\mathrm{Pic}^{0}(X)}(-D)\right|_{Z^{\prime}}\right) \rightarrow \mathrm{FM}_{\mathcal{P}}^{i}\left(\mathcal{J}_{Z}\right) \rightarrow \mathrm{FM}_{\mathcal{P}}^{i}\left(\mathcal{O}_{\mathrm{Pic}^{0}(X)}(-D)\right) \rightarrow \mathrm{FM}_{\mathcal{P}}^{i}\left(\left.\mathcal{O}_{\mathrm{Pic}^{0}(X)}(-D)\right|_{Z^{\prime}}\right) \rightarrow \ldots
$$

Since the dimension of $Z^{\prime}$ is at most $g-2$, we have $\operatorname{FM}_{\mathcal{P}}^{i}\left(\left.\mathcal{O}_{\operatorname{Pic}^{0}(X)}(-D)\right|_{Z^{\prime}}\right)=0$ for all $i>g-2$. The ampleness of $D$ (see lemma 410) and Serre duality, imply $\operatorname{FM}_{\mathcal{P}}^{i}\left(\mathcal{O}_{\operatorname{Pic}^{0}(X)}(-D)\right)=0$, for all $i \neq g$. Eventually, we conclude $\operatorname{FM}_{\mathcal{P}}\left(\mathcal{O}_{\operatorname{Pic}^{0}(X)}(-D)\right)=$ $\operatorname{FM}_{P}^{g}\left(\mathcal{J}_{Z}\right)[-g]$, and the homomorphisms $\operatorname{FM}_{P}^{g}\left(\mathcal{J}_{Z}\right) \rightarrow \operatorname{FM}_{\mathcal{P}}^{g}\left(\mathcal{O}_{\operatorname{Pic}^{0}(X)}(-D)\right)$ is an isomorphism.

The nontrivial morphism $\psi: \mathcal{J}_{Z} \rightarrow \operatorname{FM}_{\mathcal{P}}\left(\iota_{*} E\right)$ in $\mathrm{D}^{\mathrm{b}}\left(\operatorname{Pic}^{0}(X)\right)$ induces a nonzero morphism $\operatorname{FM}_{\mathcal{P}}\left(\mathcal{J}_{Z}\right)[g] \rightarrow[-1]^{*} \iota_{*} E=\operatorname{FM}_{\mathcal{P}}\left(\mathrm{FM}_{\mathcal{P}}\left(\iota_{*} E\right)\right)[g]$. The Eilenberg-Moore spectral sequence (see Theorem 2.11 in [5]) $E_{2}^{p, q}=\operatorname{Ext}^{q}\left(\operatorname{FM}_{\mathcal{P}}^{g-p}\left(\mathcal{J}_{Z}\right),[-1]^{*} \iota_{*} E\right) \Rightarrow$ $\operatorname{Ext}^{p+q}\left(\mathrm{FM}_{\mathcal{P}}\left(\mathcal{J}_{Z}\right)[g],[-1]^{*} \iota_{*} E\right)$ is a first quadrant spectral sequence. Thus, $\operatorname{Hom}\left(\mathrm{FM}_{\mathcal{P}}\left(\mathcal{J}_{Z}\right)[g],[-1]^{*} \iota_{*} E\right)=\operatorname{Hom}\left(\mathrm{FM}_{P}^{g}\left(\mathcal{J}_{Z}\right),[-1]^{*} \iota_{*} E\right)$.

Thus, we have in $\mathrm{D}^{\mathrm{b}}(\mathrm{Jac}(X))$ a commutative diagram

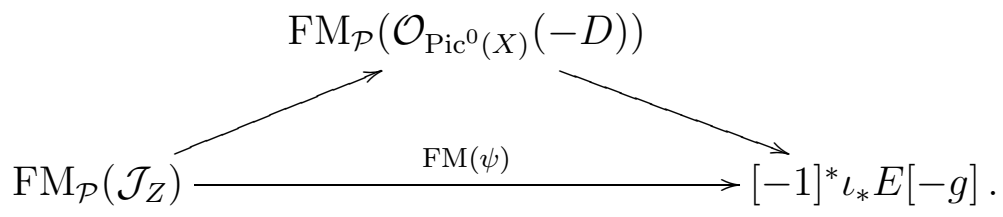

Since $[-1]^{*} \iota_{*} E[-g]=\mathrm{FM}_{\mathcal{P}}\left(\mathrm{FM}_{\mathcal{P}}\left(\iota_{*} E\right)\right)$ and $\mathrm{FM}_{\mathcal{P}}$ is an equivalence, we obtain a commu- 
tative diagram in $\mathrm{D}^{\mathrm{b}}\left(\operatorname{Pic}^{0}(X)\right)$ :

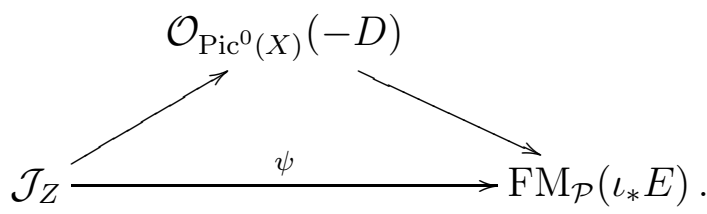

However, $H^{0}(\psi)$ is the identity of $\mathcal{J}_{Z}$. Since it factors though $\mathcal{O}_{\operatorname{Pic}^{0}(X)}(-D)$, we eventually yield the stated equality $\mathcal{J}_{Z}=\mathcal{O}_{\operatorname{Pic}^{0}(X)}(-D)$.

4.12 The vector bundle $\mathcal{E}$ on $\operatorname{Jac}(X)$. We define $\mathcal{E}$ to be the line bundle $[-1]^{*} \mathrm{FM}_{\mathcal{P}}^{g}\left(\mathcal{O}_{\operatorname{Pic}^{0}(X)}(-D)\right)$. From the morphism $\mathcal{O}_{\operatorname{Pic}^{0}(X)}(-D) \rightarrow \operatorname{FM}_{\mathcal{P}}\left(\iota_{*} E\right)$ we obtain a nontrivial morphism $\mathcal{E} \stackrel{\pi}{\longrightarrow} \iota_{*} E$.

4.13 Lemma. Properties of the vector bundle $\mathcal{E}$.

(i) The morphism $\pi: \mathcal{E} \rightarrow \iota_{*} E$ is surjective.

(ii) The rank of $\mathcal{E}$ equals $h^{0}\left(\mathcal{O}_{\operatorname{Pic}^{0}(X)}(D)\right)$;

(iii) If $[L] \in \operatorname{Pic}^{0}(\operatorname{Jac}(X))$ is the isomorphism class of a line bundle, then $\operatorname{Hom}(L, \mathcal{E})$ is of dimension one.

(iv) If $\alpha: \mathcal{E} \rightarrow k(Q)$ is a surjection to a skyscraper sheaf, then those $[L] \in \operatorname{Pic}^{0}(\operatorname{Jac}(X))$ which admit a morphism to $\operatorname{ker}(\alpha)$ form a divisor in $\operatorname{Pic}^{0}(\operatorname{Jac}(X))$.

Proof: (i) Let $G \subset \iota_{*} E$ be the image of $\pi$. The sheaf $G$ is of the form $\iota_{*} E^{\prime}$ for a subsheaf $E^{\prime} \subset E$. Since $\pi$ factors through $\iota_{*} E^{\prime}$, we have $\operatorname{FM}_{\mathcal{P}}^{0}\left(\iota_{*} E^{\prime}\right)=\mathcal{O}_{\operatorname{Pic}^{0}(X)}(-D)$. However, the minimality of $E$ implies that for all proper subsheaves $F \subset E$, we have $\operatorname{FM}_{\mathcal{P}}^{0}\left(\iota_{*} F\right)=0$. Thus, $\pi$ is surjective.

(ii) Let $Q \in \operatorname{Jac}(X)$ be a closed point. As usual, $L_{Q}$ denotes the line bundle on $\operatorname{Pic}^{0}(X)$ parameterized by $Q$. We have $\operatorname{Hom}(\mathcal{E}, k(Q))=\operatorname{Hom}\left(\mathcal{O}_{\operatorname{Pic}^{0}(X)}(-D), L_{Q}\right)=H^{0}\left(L_{Q}(D)\right)$.

(iii) Since $L=\operatorname{FM}_{\mathcal{P}}(k(R))$ for a point $R \in \operatorname{Pic}^{0}(X)$, we have

$$
\operatorname{Hom}(L, \mathcal{E})=\operatorname{Hom}\left(k(R), \mathcal{O}_{\operatorname{Pic}^{0}(X)}(-D)[g]\right)=\operatorname{Ext}^{g}\left(k(R), \mathcal{O}_{\operatorname{Pic}^{0}(X)}(-D)\right) .
$$

The sheaf $\mathcal{O}_{\operatorname{Pic}^{0}(X)}(-D)$ is a line bundle. Therefore, we have

$$
\mathcal{E}_{x t^{i}}\left(k(R), \mathcal{O}_{\operatorname{Pic}^{0}(X)}(-D)\right) \cong \begin{cases}0 & \text { for } i \neq g \\ k(R) & \text { for } i=g\end{cases}
$$

Therefore, the $H^{k}\left(\mathcal{E} x t^{l}\right) \Rightarrow$ Ext $^{k+l}$ spectral sequence degenerates, and we conclude that $\operatorname{Ext}^{g}\left(k(R), \mathcal{O}_{\operatorname{Pic}^{0}(X)}(-D)\right)=H^{0}(k(R))$ is one dimensional.

(iv) As in $(i i)$ we have $\operatorname{Hom}(\mathcal{E}, k(Q))=\operatorname{Hom}\left(\mathcal{O}_{\operatorname{Pic}^{0}(X)}(-D), L_{Q}\right)$. Thus, for any non trivial morphism $\alpha: \mathcal{E} \rightarrow k(Q)$, we obtain a nontrivial morphism $\tilde{\alpha}: \mathcal{O}(-D) \rightarrow L_{Q}$. Therefore $\tilde{\alpha}$ is injective. The Fourier-Mukai transform of $\operatorname{ker}(\alpha)$ is given by the complex $\tilde{\alpha}: \mathcal{O}(-D) \rightarrow L_{Q}$. Now the statement follows from basic facts on cohomology and base change (see II.5 in [8]).

4.14 Lemma. The surjection $\mathcal{E} \rightarrow \iota_{*} E$ factors through $\left.\mathcal{E}\right|_{C}$. Let $F$ be the kernel of $\left.\mathcal{E}\right|_{C} \rightarrow \iota_{*} E$. For the sheaf $F$ on $X$ we have $\operatorname{FM}^{0}\left(\left.\mathcal{E}\right|_{C}\right)=\operatorname{FM}_{\mathcal{P}}^{0}(F) \oplus \mathcal{O}(-D)$.

Proof: (i) From the commutative diagram

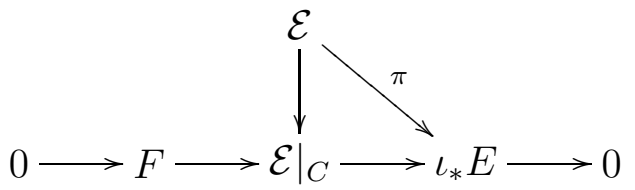


and the fact that $\pi$ induces an isomorphism $\operatorname{FM}_{\mathcal{P}}^{0}(\pi): \operatorname{FM}_{\mathcal{P}}^{0}(\mathcal{E}) \rightarrow \operatorname{FM}_{\mathcal{P}}^{0}\left(\iota_{*} E\right)$ it follows that $\operatorname{FM}_{\mathcal{P}}^{0}\left(\left.\mathcal{E}\right|_{C}\right)=\operatorname{FM}_{\mathcal{P}}^{0}(F) \oplus \operatorname{FM}_{\mathcal{P}}^{0}\left(\iota_{*} E\right)$.

\section{Base points for higher rank bundles}

5.1 Let $X$ be a smooth projective curve of genus $g \geq 2$. We say that a vector bundle $E$ with $\chi(E)=0$ has no $\Theta$-divisor in $U_{X}(R, 0)$ if for all vector bundles $F$ of rank $R$ the cohomology of $H^{\bullet}(X, E \otimes F)$ is not trivial. By the Riemann-Roch theorem $\chi(E \otimes F)=0$ implies $\operatorname{deg}(F)=0$. Furthermore from $H^{\bullet}(X, E \otimes F)=0$ we deduce the semistability of $F$. Thus, denoting the set of all semistable vector bundles of rank $R$ and degree zero on $X$ with $U_{X}(R, 0)$ we see that the above property is equivalent to

$$
\Theta_{R, E}:=\left\{F \in U_{X}(R, 0) \mid H^{\bullet}(X, E \otimes F) \neq 0\right\}=U_{X}(R, 0) .
$$

It is well known (see for example Beauville's survey articles [3] and [4]) that: if $E$ is a base point of the the $R$-th power of the generalized $\Theta$-divisor, then $E$ has no $\Theta$-divisor in $U_{X}(R, 0)$. Otherwise $\Theta_{R, E}$ is a divisor in the moduli space $U_{X}(R, 0)$.

The obvious fact that the direct sum of two semistable vector bundles $F_{1}$ and $F_{2}$ of ranks $R_{1}$ and $R_{2}$ is semistable too yields: If $E$ has a $\Theta$-divisor in $U_{X}\left(R_{1}, 0\right)$ and in $U_{X}\left(R_{2}, 0\right)$, then it has a $\Theta$-divisor in $U_{X}\left(R_{1}+R_{2}, 0\right)$.

5.2 Theorem. There exists vector bundles $P_{m, R}$ on $X$ and canonical surjections $P_{(m+1), R} \rightarrow P_{m, R}$ such that for a vector bundle $E$ of rank $r$ the conditions

(i) $E$ has no $\Theta$-divisor in $U_{X}(R, 0)$,

(ii) $\operatorname{Hom}\left(P_{m, R}, E\right) \neq 0$ for $m \gg 0$, and

(iii) $\operatorname{Hom}\left(P_{m, R}, E\right) \neq 0$ for $m>r\left(R^{2}(g-1)+1\right)$

are equivalent. The numerical invariants of the bundle $P_{m, R}$ are given by

$$
\operatorname{rk}\left(P_{m, R}\right)=R m^{\tilde{g}} \quad \operatorname{deg}\left(P_{m, R}\right)=\tilde{g} m^{\tilde{g}-1} \quad \mu\left(P_{m, R}\right)=\frac{\tilde{g}}{m R} \quad \text { with } \tilde{g}=R^{2}(g-1)+1 .
$$

Proof: Using the theory of spectral curves developed in [2] we see that there exists a finite morphism $f: \tilde{X} \rightarrow X$ of smooth projective curves of degree $R$, such that

(i) The direct image sheaf $f_{*} \mathcal{O}_{\tilde{X}}$ is given by $f_{*} \mathcal{O}_{\tilde{X}} \cong \bigoplus_{k=0}^{R-1} \omega_{X}^{\otimes-k}$. By RiemannRoch, we have $\chi\left(\mathcal{O}_{\tilde{X}}\right)=R^{2}(1-g)$. Thus, the genus $\tilde{g}$ of $\tilde{X}$ is given by $\tilde{g}=R^{2}(g-1)+1$.

(ii) Setting $\delta:=-\operatorname{deg}\left(f_{*} \mathcal{O}_{\tilde{X}}\right)=\operatorname{deg}\left(\omega_{\tilde{X}} \otimes f^{*} \omega_{X}^{-1}\right)=\left(R^{2}-R\right)(g-1)$ we obtain a dominant morphism from an open subset $U \subset \operatorname{Pic}^{\delta}(\tilde{X})$ to $U_{X}(R, 0)$ given by $\tilde{L} \mapsto f_{*} \tilde{L}$.

This is Theorem 1 of [2]. We note, that it also holds for smooth projective curves defined over an algebraically closed field of arbitrary characteristic. Since the image of $f_{*}: U \rightarrow$ $U_{X}(R, 0)$ is not contained in a proper closed subset, we conclude that $E$ has no $\Theta$-divisor in $U_{X}(R, 0)$ if and only if for all $[\tilde{L}] \in U \subset \operatorname{Pic}^{\delta}(\tilde{X})$ we have $\operatorname{Hom}\left(f_{*} \tilde{L}, E\right) \neq 0$. Since $[\tilde{L}] \mapsto \operatorname{dim}\left(\operatorname{Hom}\left(f_{*} \tilde{L}, E\right)\right)$ is upper semicontinuous we deduce that this is equivalent to $\operatorname{Hom}\left(f_{*} \tilde{L}, E\right) \neq 0$ for all $\tilde{L} \in \operatorname{Pic}^{\delta}(\tilde{X})$. The functor $E \mapsto f^{*} E \otimes \omega_{\tilde{X}} \otimes f^{*} \omega_{X}^{-1}$ is right adjoint to $\tilde{L} \mapsto f_{*} \tilde{L}$. Thus, the above condition is equivalent to $\operatorname{Hom}\left(\tilde{L}, f^{*} E \otimes \omega_{\tilde{X}} \otimes f^{*} \omega_{X}^{-1}\right) \neq 0$ for all $\tilde{L} \in \operatorname{Pic}^{\delta}(\tilde{X})$. Fixing one $\tilde{L}_{0} \in \operatorname{Pic}^{\delta}(X)$ we obtain that this is equivalent to $\operatorname{Hom}\left(\tilde{M}, \tilde{L}_{0}^{-1} \otimes f^{*} E \otimes \omega_{\tilde{X}} \otimes f^{*} \omega_{X}^{-1}\right) \neq 0$ for all $\tilde{M} \in \operatorname{Pic}^{0}(\tilde{X})$. Now we can apply our Theorem 25] to deduce, that this is equivalent to $\operatorname{Hom}\left(\tilde{P}_{m}, \tilde{L}_{0}^{-1} \otimes f^{*} E \otimes \omega_{\tilde{X}} \otimes f^{*} \omega_{X}^{-1}\right) \neq 0$ for $m \gg 0$ with $\tilde{P}_{m}$ the Raynaud bundle on $\tilde{X}$. As before, we conclude that this is 
equivalent to $\operatorname{Hom}\left(f_{*}\left(\tilde{P}_{m} \otimes \tilde{L}_{0}\right), E\right) \neq 0$ for $m \gg 0$. Thus setting $P_{m, R}:=f_{*}\left(\tilde{P}_{m} \otimes \tilde{L}_{0}\right)$ we have shown the equivalence of (i) and (ii). Applying Theorem 317 instead of Theorem 25 we obtain the equivalence of (i) and (iii). The computation of the numerical invariants is straightforward.

\section{Applications and questions}

\subsection{Elliptic curves}

6.1 Vector bundles on elliptic curves. Let $X$ be an elliptic curve. In this case we have (see Lemma 231) the numerical invariants $\operatorname{rk}\left(P_{m}\right)=m$, and $\operatorname{deg}\left(P_{m}\right)=1$. Therefore, the slope $\mu\left(P_{m}\right)=\frac{1}{m}$ is always positive.

Thus, for a vector bundle $E$ of rank $r$ and degree 0 we deduce the following equivalences $E$ is semistable $\Leftrightarrow \operatorname{Hom}\left(P_{r+1}, E\right)=0 \quad \Leftrightarrow \quad H^{*}(X, E \otimes L)=0$ for a $L \in \operatorname{Pic}^{0}(X)$.

Indeed, if $E$ is semistable, then from $\mu\left(P_{r+1}\right)=\frac{1}{r+1}>0=\mu(E)$ we derive that $\operatorname{Hom}\left(P_{r+1}, E\right)=0$. However, $\operatorname{Hom}\left(P_{r+1}, E\right)=0$ implies by Theorem 317 that $E$ has a $\Theta$-divisor. Having a $\Theta$-divisor implies semi stability immediately.

Moreover, Polishchuk shows in [9] that the Fourier-Mukai transform $\mathrm{FM}_{\mathcal{P}}$ gives an equivalence between semistable bundles of rank $r$ and degree zero and torsion sheaves of length $r$. See [6] for a presentation of Atiyah's results on vector bundles on elliptic curves (cf. [1]) in terms of Fourier-Mukai transforms.

\subsection{Curves of genus two}

In this subsection we assume $X$ to be a curve of genus two defined over the complex numbers. Recall that from 42 and 43 that $r_{-}(X)$ is the smallest rank of a stable vector bundle $E$ on $X$ with $\operatorname{Hom}\left(P_{m}, E\right) \neq 0$ for $m \gg 0$ and $\mu(E) \leq g-1$.

6.2 Theorem. If $X$ is a curve $X$ of genus 2 , then $r_{-}(X)=4$.

Proof: By 23 the Raynaud bundle $P_{2}$ is of rank four and has $\mu\left(P_{2}\right)=1$. Thus, we have to exclude the ranks one, two, and three as possible ranks of a stable vector bundle $E$ with $\operatorname{Hom}\left(P_{m}, E\right) \neq 0$ and $\mu(E) \leq 1$.

Case 1: $\operatorname{rk}(E)=1$.

This case can easily excluded because line bundles of degree $d \leq g-1$ have a $\Theta$-divisor.

Case 2: $\operatorname{rk}(E)=2$.

Here we have two subcases depending of the parity of the degree of $E$.

Case 2.1: $\operatorname{rk}(E)=2$ and $\operatorname{deg}(E)$ is even.

Let $D$ be an effective divisor of degree $\frac{2 g-2-\operatorname{deg}(E)}{2}$ and take a global section $\mathcal{O}_{X} \stackrel{s}{\longrightarrow} \mathcal{O}_{X}(D)$. This way we obtain a semistable vector bundle $E(D)=E \otimes \mathcal{O}_{X}(D)$ with $\mu(E(D))=g-1$, and the embedding $E \rightarrow E(D)$ gives $\operatorname{Hom}\left(P_{m}, E(D)\right) \neq 0$. Thus $E(D)$ is a base point for the generalized $\Theta$-divisor which is impossible for vector bundles of rank two as shown by Raynaud in [10] Corollaire 1.7.4.

Case 2.2: $\operatorname{rk}(E)=2$ and $\operatorname{deg}(E)$ is odd.

Take an extension $0 \rightarrow E \rightarrow E^{\prime} \rightarrow k\left(x_{0}\right) \rightarrow 0$ with $E^{\prime}$ a vector bundle. Since any subsheaf $L \subset E^{\prime}$ gives a subsheaf $L\left(-x_{0}\right)$ of $E$, the stability of $E$ implies the semistability of $E^{\prime}$. Thus, we pass to a semistable vector bundle $E^{\prime}$ with $\operatorname{deg}\left(E^{\prime}\right)$ even and $\operatorname{Hom}\left(P_{m}, E^{\prime}\right) \neq 0$. Now we proceed as in case 2.1. 
Case 3: $\operatorname{rk}(E)=3$.

Here we have three subcases according to $\operatorname{deg}(E)$ modulo 3 .

Case 3.1: $\operatorname{rk}(E)=3$ and $\operatorname{deg}(E) \equiv 0 \bmod 3$.

Analogously to case 2.1 we obtain a base point for the generalized $\Theta$-divisor on rank three bundles which contradicts again Raynaud's result.

Case 3.2: $\operatorname{rk}(E)=3$ and $\operatorname{deg}(E) \equiv-1 \bmod 3$. We proceed as in case 2.2.

Case 3.3: $\operatorname{rk}(E)=3$ and $\operatorname{deg}(E) \equiv 1 \bmod 3$.

Here we take a short exact sequence $0 \rightarrow E^{\prime} \rightarrow E \rightarrow k\left(x_{0}\right) \rightarrow 0$. The stability of $E$ implies as before the semistability of $E^{\prime}$. However $E$ is contained in $E^{\prime}\left(x_{0}\right)$. The latter is semistable of degree divisible by three. Hence case 3.1 applies.

6.3 Corollary. On a curve of genus 2 the Raynaud bundle $P_{2}$ is stable.

Proof: We have $\mu\left(P_{2}\right)=1$ by 23. Let $P_{2} \rightarrow E$ be the surjection to a stable bundle with $\mu(E) \leq 1$. By the above Theorem 62 we have $\operatorname{rk}(E)=4$.

\subsection{Further examples}

6.4 Curves of genus $g \geq 3$. Suppose that $X$ is a curve of genus 3 defined over the complex numbers. Even though we can copy the proof of Theorem 62 we only obtain $r_{-}(X) \geq 4$. Here we use the base point freeness of the generalized $\Theta$-divisor of bundles of rank two and three on the curve $X$ (see Proposition 1.6 in [4]).

Since base point freeness of the generalized $\Theta$-divisor on the moduli space of rank three bundles is not known for a curve $X$ of genus $g \geq 4$ we obtain a priori only $r_{-}(X) \geq 3$.

6.5 Quot-schemes without torsion quotients. Let $X$ be a curve of positive genus $g$, and $m>g$ an integer. Since any line bundle $L_{0}$ of Euler characteristic zero has a $\Theta$-divisor, we conclude $\operatorname{Hom}\left(P_{m}, L_{0}\right)=0$. At the other hand we have $\operatorname{Hom}\left(P_{m}, L_{1}\right) \neq 0$ for line bundles of positive Euler characteristic by Lemma 36.

If $\psi: P_{m} \rightarrow L_{1}$ is a non-trivial morphism, then it must be surjective. Otherwise it would factor through a subsheaf $L_{1}(-D)$ for a non-trivial effective divisor $D$. This contradicts the fact, that there exists no homomorphism from $P_{m}$ to a line bundle of Euler characteristic zero. We conclude that the Quot-scheme $\operatorname{Quot}_{X}^{1, g}\left(P_{m}\right)$ of rank one quotients of degree $g$ of $P_{m}$ has no torsion quotients. On the other hand, every line bundle $L_{1}$ of degree $g$ appears as a quotient of $P_{m}$ by Lemma 36.

6.6 Quot-schemes parameterizing only stable quotients. Let $X$ be a curve of genus $g \geq 2$. The number $r_{1}$ is the smallest possible rank for a $\chi$-small vector bundle $E$ with $\operatorname{Hom}\left(P_{m}, E\right) \neq 0$ for $m \gg 0$. Let $d_{-}$be the smallest possible degree of such a bundle.

Then for $m \gg 0$ the Quot scheme $\operatorname{Quot}_{X}^{r_{-}, d_{-}}\left(P_{m}\right)$ of quotients of $P_{m}$ of rank $r_{-}$and degree $d_{-}$parameterizes only $\chi$-small vector bundles. Indeed, if $E$ is not semistable, then it contains a subsheaf $E_{1} \subset E$ of maximal slope. The surjection of $P_{m} \rightarrow E \rightarrow E / E_{1}$ gives us a surjection of $P_{m}$ to $E^{\prime \prime}=E / E_{1}$ with $r k\left(E^{\prime \prime}\right)<\operatorname{rk}(E)$ and $\operatorname{deg}\left(E^{\prime \prime}\right)<\operatorname{deg}(E)$. Proceeding this way we obtain a surjection from $P_{m}$ to a $\chi$-small bundle of degree less than $r_{-}$which is impossible by the very definition of $r_{-}$. Thus, each quotient of $P_{m}$ with these numerical invariants is a stable vector bundle. By definition of $r_{-}$and $d_{-}$the scheme $\operatorname{Quot}_{X}^{r_{-}, d_{-}}\left(P_{m}\right)$ is not empty for $m \gg 0$.

6.7 Generalized Raynaud bundles. For an ample line bundle $L$ on $\operatorname{Pic}^{0}(X)$ we can define

$$
P_{L}:=\iota^{*}[-1]_{\operatorname{Jac}(X)}^{*} R^{g} q_{*}\left(\mathcal{P} \otimes p^{*} L^{-1}\right)
$$


having the definition of the bundles $P_{m}$ in mind we obtain $P_{m}=P_{m \cdot \Theta}$. Indeed, theorem 46 implies that to each minimal bundle $E$ there exists a unique ample divisor $D(E)$ on $\operatorname{Pic}^{0}(X)$ with a unique (up to scalars) surjection $P_{D} \rightarrow E$.

However, we gave another generalization of Raynaud's vector bundles with the bundles $P_{m, R}$ of theorem [52] To unify both, we consider a morphism $\pi: \tilde{X} \rightarrow X$ of irreducible smooth curves such that $\pi_{*} \mathcal{O}_{\tilde{X}} \cong \oplus_{k=0}^{R-1} \omega_{X}^{\otimes-k}$, and a line bundle $\tilde{L}_{0}$ on $\tilde{X}$ of degree $\delta=$ $\left(R^{2}-R\right)(g-1)$. Now any vector bundle $E$ with

- $\operatorname{Hom}(F, E) \neq 0$ for all vector bundles $F$ of $\operatorname{rank} R$ with $\chi(F)=0$; and

- For all proper subsheaves $E^{\prime} \subset E$, there exists a rank $R$ vector bundle $F$ with $\chi(F)=0$ and $\operatorname{Hom}\left(F, E^{\prime}\right)=0$.

determines an ample divisor $\tilde{D}$ in the Picard group $\operatorname{Pic}^{0}(\tilde{X})$ and a surjection $\pi_{*}\left(P_{\tilde{D}} \otimes \tilde{L}_{0}\right) \rightarrow$ $E$. To proof this claim just combine the theorems 46] and 52 .

Having said this, it is natural to consider the vector bundles $P_{\tilde{D}, R}:=\pi_{*}\left(P_{\tilde{D}} \otimes \tilde{L}_{0}\right)$ with $\tilde{D}$ an ample divisor on $\operatorname{Pic}^{0}(\tilde{X})$ as the generalized Raynaud bundles.

6.8 Base points of the generalized $\Theta$-divisor. To study the base points of the generalized $\Theta$-divisor on $\mathrm{U}(r, r(g-1))$ it is by theorem 37 enough to study all quotients $Q$ of $P_{r g+1}$. Indeed, any base point corresponding to the sheaf $E$ contains by this theorem a quotient $Q$ of $P_{r g+1}$ with (semistability of $E$ ) Euler characteristic $\chi(Q) \leq 0$. The sheaves $P_{m}$ with $1<m<g r+1$ are quotients of this type. See [1] for such a construction.

6.9 Question: Does $r_{-}(X)$ varies with $X$ ? It seems to me very probable that a curve $X$ where the Néron-Severi group $\mathrm{NS}\left(\mathrm{Pic}^{0}(X)\right)$ has high rank should have a smaller $r_{-}(X)$ than a curve $X^{\prime}$ with rank of $\mathrm{NS}\left(\operatorname{Pic}^{0}\left(X^{\prime}\right)\right)$ equal to one.

It is my hope that the generalized Raynaud bundles of 67 will help to understand the dependence of $r_{-}(X)$ from the Néron-Severi group $\mathrm{NS}\left(\operatorname{Pic}^{0}(X)\right)$. 


\section{References}

[1] M. F. Atiyah, Vector bundles over an elliptic curve, Proc. London Math. Soc. 7 (1957) 414-452.

[2] A. Beauville, M. S. Narasimhan, S. Ramanan, Spectral curves and the genralised theta divisor, J. reine angew. Math. 398 (1989) 169-179.

[3] A. Beauville, Vector bundles on curves and generalized theta functions: recent results and open problems, in Current Topics in Complex Algebraic Geometry, MSRI Publ. 28 (1995) 17-33.

[4] A. Beauville, Vector bundles on curves and theta functions, 2005, math. AG/0502179.

[5] A.I. Bondal, M.M. Kapranov, Representable functors, Serre functors, and reconstructions, Math. USSR Izv. 35 (1990) 519-541.

[6] G. Hein and D. Ploog, Stable bundles and Fourier-Mukai transforms on elliptic curves, Contributions to Algebra and Geometry 46 Vol. 2 (2005) 423-434.

[7] S. Mukai, Duality between $\mathrm{D}(X)$ and $\mathrm{D}(\hat{X})$ and its application to Picard sheaves, Nagoya Math. J. 81 (1981) 153-175.

[8] D. Mumford, Abelian Varieties, Oxford University Press, Oxford, 1970.

[9] A. Polishchuk, Abelian varieties, Theta functions and the Fourier Transform, Cambridge University Press, Cambridge UK, 2003.

[10] M. Raynaud, Section des fibrés vectoriels sur une courbe, Bull. Soc. math. France 110 (1982) 103-125.

[11] O. Schneider, Sur la dimension de l'ensemble des points base du fibré déterminant sur $\mathrm{SU}_{C}(r), 2005$, math.AG/0501320.

Georg Hein, Universität Duisburg-Essen, Fachbereich Mathematik, 45117 Essen email: georg.hein@uni-due.de 